\title{
Cancer risk in patients with Noonan syndrome carrying a PTPN11 mutation
}

\author{
Marjolijn CJ Jongmans ${ }^{\star, 1}$, Ineke van der Burgt ${ }^{1}$, Peter M Hoogerbrugge ${ }^{2}$, Kees Noordam ${ }^{3}$, Helger G Yntema ${ }^{1}$, \\ Willy M Nillesen ${ }^{1}$, Roland P Kuiper ${ }^{1}$, Marjolijn JL Ligtenberg ${ }^{1}$, Ad Geurts van Kessel ${ }^{1}$, J Han JM van Krieken ${ }^{4}$, \\ Lambertus ALM Kiemeney ${ }^{5,6}$ and Nicoline Hoogerbrugge ${ }^{1}$
}

Noonan syndrome (NS) is characterized by short stature, facial dysmorphisms and congenital heart defects. PTPN11 mutations are the most common cause of NS. Patients with NS have a predisposition for leukemia and certain solid tumors. Data on the incidence of malignancies in NS are lacking. Our objective was to estimate the cancer risk and spectrum in patients with NS carrying a PTPN11 mutation. In addition, we have investigated whether specific PTPN11 mutations result in an increased malignancy risk. We have performed a cohort study among 297 Dutch NS patients with a PTPN11 mutation (mean age 18 years). The cancer histories were collected from the referral forms for DNA diagnostics, and by consulting the Dutch national registry of pathology and the Netherlands Cancer Registry. The reported frequencies of cancer among NS patients were compared with the expected frequencies using population-based incidence rates. In total, 12 patients with NS developed a malignancy, providing a cumulative risk for developing cancer of $23 \%$ (95\% confidence interval (Cl), 8-38\%) up to age 55 years, which represents a 3.5 -fold $(95 \% \mathrm{Cl}, 2.0-5.9)$ increased risk compared with that in the general population. Hematological malignancies occurred most frequently. Two malignancies, not previously observed in NS, were found: a malignant mastocytosis and malignant epithelioid angiosarcoma. No correlation was found between specific PTPN11 mutations and cancer occurrence. In conclusion, this study provides first evidence of an increased risk of cancer in patients with NS and a PTPN11 mutation, compared with that in the general population. Our data do not warrant specific cancer surveillance. European Journal of Human Genetics (2011) 19, 870-874; doi:10.1038/ejhg.2011.37; published online 16 March 2011

Keywords: Noonan syndrome; PTPN11 gene; cancer incidence; cumulative risk

\section{INTRODUCTION}

Noonan syndrome (NS, OMIM 163950) is an autosomal dominant developmental disorder characterized by short stature, facial dysmorphisms and congenital heart defects (reviewed in Tartaglia et al ${ }^{1}$ ). It is a relatively common syndrome with an estimated incidence of 1 in 2500 births. NS is associated with a higher risk for benign and malignant proliferative disorders. Approximately $10 \%$ of the children with NS are affected by a mild myeloproliferative disorder, which is usually self-limiting. ${ }^{2}$ A smaller percentage of patients develops juvenile myelomonocytic leukemia (JMML) ${ }^{3,4}$ and/or other hematological malignancies. ${ }^{5-11}$ In addition, several case reports have suggested a possible increased incidence of solid tumors in NS patients, specifically neuroblastoma and embryonal rhabdomyosarcoma. ${ }^{12-19}$ Benign proliferative conditions reported include multiple giant cell lesions and granular cell tumors. ${ }^{20,21}$ Until now, heterozygous germline mutations in six genes encoding components of the RAS/MAPK pathway, that is, PTPN11, KRAS, SOS1, RAF1, NRAS and SHOC2 have been identified in NS. ${ }^{1}$ The RAS/MAPK pathway represents a signal transduction cascade involved in processes of cell proliferation, differentiation, survival and death. Mutations in PTPN11, which encodes the non-receptor protein tyrosine phosphatase SHP-2, are most frequently observed and account for $\sim 50 \%$ of NS cases. SHP-2 has two Src homology 2 domains
(N-SH2 and C-SH2), a catalytic protein tyrosine phosphatase (PTP) domain and a $\mathrm{COOH}$ terminus containing tyrosine phosphorylation sites. Most PTPN11 mutations involved in NS disrupt the autoinhibitory interaction between the N-SH2 and the PTP domain, resulting in enhanced phosphatase activity and increased signaling through the RAS-MAPK pathway. ${ }^{22}$

The observed increase in JMML incidence in NS and the important role of SHP-2 in RAS signaling, suggest a role of mutant SHP-2 in cancer development. Indeed, somatic mutations in PTPN11 occur in $\sim 35 \%$ of sporadic JMML and in a small percentage of other hematological malignancies. ${ }^{23-25}$ Similarly, solid tumors have been screened for somatic PTPN11 mutations. In some of them, such as lung-, liver- and colorectal cancer, PTPN11 mutations have been identified, be it infrequent. ${ }^{19,26}$ On the basis of these findings, and considering the universal role of increased RAS-MAPK signaling in oncogenesis, we hypothesized that patients with NS might have an increased risk for developing a broad range of malignancies, in addition to leukemia. So far, no quantitative data are available on the risk of cancer in NS caused by PTPN11 mutations. Therefore, guidelines for cancer prevention in patients with NS are yet to be developed. We have performed a first cohort study to explore the cumulative risk of developing cancer in patients with NS carrying a

\footnotetext{
${ }^{1}$ Department of Human Genetics, Radboud University Nijmegen Medical Center, Nijmegen, The Netherlands; ${ }^{2}$ Departments of Pediatric Hemato-oncology, Radboud University Nijmegen Medical Center, Nijmegen, The Netherlands; ${ }^{3}$ Department of Pediatric Endocrinology, Radboud University Nijmegen Medical Center, Nijmegen, The Netherlands; ${ }^{4}$ Department of Pathology, Radboud University Nijmegen Medical Center, Nijmegen, The Netherlands; ${ }^{5}$ Departments of Epidemiology, Biostatistics and HTA, Radboud University Nijmegen Medical Center, Nijmegen, The Netherlands; ${ }^{6}$ Department of Urology, Radboud University Nijmegen Medical Center, Nijmegen, The Netherlands

*Correspondence: Dr MCJ Jongmans, Department of Human Genetics, Radboud University Nijmegen Medical Center, PO Box 9101,6500 HB Nijmegen, The Netherlands. Tel: +31 24 3613946; Fax: +31 24 3668774; E-mail: m.jongmans@antrg.umcn.nl

Received 6 October 2010; revised 4 January 2011; accepted 4 February 2011; published online 16 March 2011
} 
germline PTPN11 mutation. In addition, we investigated whether specific PTPN11 mutations result in an increased cancer risk and whether specific malignancies are particularly associated with NS. For the latter purpose, we have reviewed all NS patients with a PTPN11 mutation and a malignancy reported in the literature.

\section{PATIENTS AND METHODS}

\section{Study population}

The study population consisted of 318 Dutch patients with NS in whom a germline PTPN11 mutation was identified in the laboratory for DNA diagnostics of the Department of Human Genetics, Radboud University Nijmegen Medical Center, the Netherlands. To obtain a genetically uniform cohort of patients, we exclusively included patients with a PTPN11 mutation. This gene is most commonly involved in NS, accounting for $\sim 50 \%$ of patients. The mutations were identified between January 2002 and December 2008. Patients with a mutation related to LEOPARD syndrome $(n=17)$ and patients who died prenatally or in the first 3 months of life $(n=4)$ were excluded. After this exclusion, the cohort consisted of 235 index patients and 62 affected family members, of which 143 (48\%) were males and 154 (52\%) were females. At the end of the follow-up period, the patients had a mean age of 18 years and a median age of 13 years (range 0.6-95 years).

\section{Cancer follow-up}

Diagnoses of cancer were retrieved using three different sources of information. First, we screened the medical history of the patients using the referral forms for PTPN11 analysis. Next, we performed a search in the National Automated Archive of Pathology (PALGA). All histology and cytology reports in the Netherlands are collected in this national database, which was founded in 1971 and achieved virtually complete national coverage in $1980 .{ }^{27}$ As PALGA is an incomplete database for hematological malignancies and lacks malignancies without histological confirmation, we also consulted The Netherlands Cancer Registry (NCR). The NCR has a complete national coverage since 1989.

\section{Statistical methods}

Time at risk started on date of birth of the patient and ended on the date of cancer diagnosis, emigration, the date of death or the closing date of the study (5 December 2008), whichever occurred first. Cumulative risks of cancer were calculated by standard Kaplan-Meier analysis in which the end point was defined as a diagnosis of any cancer and follow-up was defined as age at diagnosis. Patients who died before a cancer diagnosis, were lost to follow-up or reached the end of the study period without a cancer diagnosis, were censored at the corresponding age.

To be able to compare the observed cumulative risk of cancer among the study population with the average cumulative risk in the Dutch population, we calculated the expected frequency of cancer in the study population based on the Dutch population-based incidence rates in 2002-2006 http://www. ikcnet.nl). Because the Netherlands has a nationwide population-based cancer registry, reference values can be taken efficiently from the registry and can be considered more valid and stable than estimates taken from a specific (smaller) control group. Basal cell carcinoma of the skin was excluded from the analysis, as this neoplasm is not registered in the NCR. For the calculation of the expected frequency, we multiplied the gender and 5-year age-specific population years in the study population with the gender and 5-year age-specific incidence rates in the Dutch population. Subsequently, the 95\% confidence intervals (CIs) of the observed versus expected ratios were calculated using the open source program OpenEpi version 2.3 (http://www.openepi.com).

\section{Sequence analyses}

Sequencing of PTPN11 exons 1-4, 7, 8 and 11-14 and surrounding splice donor and acceptor sites was performed in a routine DNA diagnostic setting (www.dnadiagnostieknijmegen.nl) on DNA isolated from peripheral blood cells. Primer sequences and PCR conditions are available upon request. In one patient who was diagnosed with acute lymphocytic leukemia (ALL), mutation analysis was repeated on DNA isolated from gastric antrum tissue, to confirm the germline status of the mutation.

Except for one JMML and one malignant mastocytosis, all malignancies were available for sequence analysis. In the patient with ALL, DNA was extracted from bone marrow cells, in all other cases DNA was extracted from paraffin-embedded tumor tissue. The sample derived from the patient with basal cell carcinoma, had a tumor cell percentage of less than $60 \%$ and was, therefore, excluded from further analysis.

Primer pairs were developed that covered a region of approximately 150 base pairs, including the PTPN11 mutation identified in the germline of the patient. Primer sequences and PCR conditions are available upon request.

\section{RESULTS}

The median follow-up time for cancer in our cohort of 235 index patients and 62 affected family members was 13 years (range 0.6-95 years). Approximately $18.5 \%$ of the patients were followed for less than 5 years, $36.4 \%$ for $5-15$ years and $45.1 \%$ for more than 15 years. In total, 12 malignancies were observed: three in children ( $\leq 15$ years), three in adolescents and young adults (15-29 years) and six in adults ( $\geq 30$ years) (Table 1 ). The median age at diagnosis was 25 years (range: 0-57). One patient developed a basal cell carcinoma that was not included in our statistical analyses. In one case, the definitive cancer diagnosis was unclear and, as a consequence, this patient was censored at the date of that arbitrary diagnosis. This latter patient, a 5-year-old child, carried a c.188A $>$ G (p.Tyr63Cys) PTPN11 mutation and was diagnosed with nephroblastomatosis and, possibly, a small nephroblastoma. The germline status of the c.188A $>\mathrm{G}$ mutation in the patient with ALL was confirmed through the analysis of DNA isolated from previously obtained stomach (gastric antrum) tissue from this patient. Out of 10, 5 PTPN11 mutations found in our NS patients with a malignancy were previously reported as somatic mutations in sporadic malignancies (Table 2). The frequency of malignancies among carriers of the same germline mutation in our cohort is also displayed in Table 2.

Assessment of the occurrence of cancer in our study cohort (Figure 1) provided a cumulative risk of $23 \%(95 \% \mathrm{CI}, 8-38 \%)$ up to the age of 55 years, which represents a 3.5 -fold increased risk $(95 \%$ CI, 2.0-5.9) compared with that in the general population.

Breast cancer was observed twice, whereas all other malignancies were observed only once. Six cancer types have previously been reported in the literature in patients with NS and a PTPN11 mutation, that is, JMML, neuroblastoma, ALL, non-Hodgkin lymphoma, glioma and breast cancer (Table 1).

The child with JMML harbored a de novo p.Gly503Ala mutation. To our knowledge, this mutation has not yet been reported as a germline mutation in a patient with NS, but only as a somatic event in JMML. ${ }^{23,24,28,29}$ Other tissues of this patient were unavailable to confirm the germline status of the mutation. However, the child was reported to have a valvular pulmonic stenosis, an atrial septum defect, hypertelorism and low-set ears with a thick helix, and, therefore, likely had NS. Photographs of the patient were seen by Dr I van der Burgt (coauthor), and she considered the patient's facial features as suggestive for NS. It can be difficult to distinguish a benign myeloproliferative disorder, which is usually self-limiting and frequently observed in NS, from the rare aggressive condition JMML. ${ }^{28,30,31}$ Assessment of the clonal status of the monocytic population can be helpful in this respect, ${ }^{32}$ but we were unable to infer whether such a diagnostic test was performed in this patient. The patient underwent a bone marrow transplantation, indicating that the course of the disease was aggressive.

In a search for copy number alterations of PTPN11 mutant alleles, we performed sequence analysis in tumor DNA of 9 out of 12 patients diagnosed with a malignancy. None of the sequences showed an overrepresentation of the mutated allele compared with the wildtype allele. 
Table 1 Patients with Noonan syndrome carrying a germline PTPN11 mutation and diagnosed with a malignancy reported here (1-12) and from the literature

\begin{tabular}{|c|c|c|c|}
\hline No./Ref. & Malignancy & Age at diagnosis & PTPN11 mutation \\
\hline 1 & JMML & 1 year & c. $1508 \mathrm{G}>\mathrm{C}$ (p.Gly503Ala) \\
\hline 2 & Neuroblastoma (adrenal gland) & 1 year & c. $844 \mathrm{~A}>\mathrm{G}$ (p.lle282Val) \\
\hline Mutesa et $a l^{18}$ & Neuroblastoma & 5 months & c.179G > C (p.Gly60Ala) \\
\hline Bentires-Alj et a/ ${ }^{19}$ & Neuroblastoma & $?$ & c. 185 A > C (p.Tyr62Cys) \\
\hline Kondoh et al16 & Neuroblastoma & 6 months & c. $1504 T>A$ (p.Ser502Thr) \\
\hline Chantrain et $a F^{5}$ & Neuroblastoma ${ }^{a}$ & 4 years & c. $922 A>G(p . A s n 308 A s p)$ \\
\hline 3 & Dysembryoplastic neuro-epithelial tumor (temporal lobe) & 10 years & c.179G > C (p.Gly60Ala) \\
\hline 4 & Oligodendroglioma (hypothalamus) ${ }^{\mathrm{b}}$ & 17 years & c.417G > C (p.Glu139Asp) \\
\hline Sherman et $a^{\beta 3}$ & Low grade glioneural tumor & 6 years & c. $172 A>G$ (p.Asn58Asp) \\
\hline Martinelli et $a^{\beta 4}$ & Oligodendrogliomac & 24 years & c. $64 A>G$ (p.Thr22Ala) \\
\hline Schuettpelz et $a^{\beta 5}$ & Pilocytic astrocytoma & 8 years & c.1471_1472delinsTT (p.Pro491Phe) \\
\hline 5 & Precursor B-ALL & 23 years & c.188A > G (p.Tyr63Cys) \\
\hline Roti et al ${ }^{11}$ & Common B ALL & 17 years & c.179G >C (p.Gly60Ala) \\
\hline Karow et $a l^{7}$ & Pre-B cell ALL & 2 years & c. $1510 A>G$ (p.Met504Val) \\
\hline Karow et al & Pre-B cell ALL & 8 years & c. $417 G>C$ (p.Glu139Asp) \\
\hline Chantrain et $a F^{5}$ & AML-M4 ${ }^{a}$ & 5 years & c. $922 A>G(p . A s n 308 A s p)$ \\
\hline La Starza et a $\beta$ & AML-M4 & 34 years & c.178G > T (p.Gly60Cys) \\
\hline Matsubara et al ${ }^{10}$ & CMML & 35 years & c. $922 A>G(p . A s n 308 A s p)$ \\
\hline 6 & Non-Hodgkin lymphoma (jejunum) & 25 years & c. $1472 C>T$ (p.Pro491Leu) \\
\hline Ferrero et a/6 & Non-Hodgkin lymphomad & $?$ & c. $1508 \mathrm{G}>$ A (p.Gly503Glu) \\
\hline Lo et a ${ }^{\rho}$ & Hodgkin's lymphoma & 35 years & c. $1507 G>C$ (p.Gly503Arg) \\
\hline 7 & Multifocal ductal breast cancer & 48 years & c.774G > T (p.Glu258Asp) \\
\hline 8 & Ductal breast cancer & 52 years & c.925A > G (p.lle309Val) \\
\hline Shaw et $a^{\beta 6}$ & Breast cancere & 45 years & c.317A >C (p.Asp106Ala) \\
\hline 9 & Malignant epithelioid angiosarcoma (mediastinum) & 38 years & c. $1510 A>G$ (p.Met504Val) \\
\hline 10 & Colorectal cancer (sigmoid) & 44 years & c. $1510 A>$ G (p.Met504Val) \\
\hline 11 & Malignant mastocytosis & 46 years & c.922A > G (p.Asn308Asp) \\
\hline 12 & Basal cell carcinoma & 57 years & c. 188 A > G (p.Tyr63Cys) \\
\hline Yoshida et $a^{\beta 7}$ & Hepatoblastoma & 1 months & c. $922 A>G(p . A s n 308$ Asp) \\
\hline
\end{tabular}

The lines in white are patients from our study cohort, the lines in gray are patients reported in the literature. Reports of children with NS who developed JMML are not included, these are summarized in the report of Aoki et al. ${ }^{38}$

asame patient.

${ }^{b} T h i s$ patient has previously been published by us. ${ }^{39}$

cThe p.Thr22Ala change was found in a brain tumor and subsequently in peripheral blood cells. The patient had no features of NS. This molecular lesion results in an increased basal phosphatase activity of PTPN11 and was not observed in over 300 control individuals. ${ }^{34}$

dThe mutation was identified in the son of this patient. The mother had short stature and a facial appearance typical of NS. DNA was not available for segregation analysis. eMutation details derived by personal communication with the author.

\section{DISCUSSION}

Our cohort study shows a 3.5-fold (95\% CI, 2.0-5.9) increase in the overall cancer risk up to the age of 55 years in NS patients with a PTPN11 mutation, compared with that in the general population. We encountered six patients who developed cancer at adult age, whereas until now only four adult patients with a germline PTPN11 mutation and a malignancy have been reported in the literature. The overall risk of cancer up to the age of 70 years cannot be estimated because of the age distribution in our cohort, with a majority of patients below 30 years of age.

As expected, hematological malignancies were most frequent (4/12, $33 \%)$. In one patient, this malignancy was specified to be a malignant mastocytosis, which so far has not been reported in NS. The nonhematological malignancies observed included three novel cancers in NS: a malignant epithelioid angiosarcoma, a colon cancer of the sigmoid and a basal cell carcinoma.

One way to explore potential correlations between germline PTPN11 mutations and the development of a specific malignancy is screening for loss of heterozygosity and/or uniparental disomy, in conjunction with duplication or amplification of a heterozygous germline PTPN11 mutation in the tumors of patients. This two-hit scenario was previously observed in NS, but is not a consistent finding in all NS-associated malignancies. ${ }^{7,40}$ In our cohort, we did not observe multiple copies of the mutant PTPN11 allele in nine tumors tested. As a consequence, we can only speculate about the influence of PTPN11 mutations on the development of these malignancies. For instance, we found one patient with colorectal cancer diagnosed at an unusually young age of 44 years. This early onset may be related to the PTPN11 mutation. In sporadic colorectal cancer an association has been found with the RAS-MAPK pathway and activating mutations in the KRAS oncogene are known to have a role in the progression from a benign adenoma to a dysplastic adenocarcinoma. ${ }^{41}$ Nearly $50 \%$ of colon cancers harbor activating mutations in KRAS. ${ }^{41}$ Therefore, the germline PTPN11 mutation in the patient with colorectal cancer may have played a role in tumor progression.

Similarly, a relation between PTPN11 and breast cancer has not been noted before. Remarkably, however, two patients in our cohort (48 and 52 years of age) developed breast cancer, which has a mean age at diagnosis of 60 years in the general population. Interestingly, several studies have shown a role for RAS-MAPK pathway activation in breast cancer development. ${ }^{42}$ Furthermore, women with Neurofibromatosis type1, another syndrome associated with aberrant RAS 
Table 2 Frequency of PTPN11 mutations associated with cancer in our cohort and literature data on its somatic occurrence

\begin{tabular}{llc}
\hline PTPN11 mutation & $\begin{array}{c}\text { Cancer related } \\
\text { frequency }^{2}\end{array}$ & $\begin{array}{c}\text { Previously reported as somatic } \\
\text { mutation in cancer }\end{array}$ \\
\hline c.179G $>$ C (p.Gly60Ala) & 1 of 8 & + \\
c.188A $>$ G (p.Tyr63Cys) & 2 of 41 & + \\
c.417G $>$ C (p.Glu139Asp) & 1 of 17 & + \\
c.774G $>$ T (p.Glu258Asp) & 1 of 3 & - \\
c.844A $>$ G (p.lle282Val) & 1 of 14 & - \\
c.922A $>$ G (p.Asn308Asp) & 1 of 62 & - \\
$c .925 A>G$ (p.lle309Val) & 1 of 3 & + \\
$c .1472 C>T$ (p.Pro491Leu) & 1 of 4 & + \\
$c .1508 G>C$ (p.Gly503Ala) & 1 of 1 & - \\
$c .1510 A>G$ (p.Met504Val) & 2 of 17 &
\end{tabular}

aThe second number represents how often the mutation has been identified in our cohort, whereas the first number displays the number of patients in the cohort with this mutation who developed a malignancy.

bThe data in this column are derived from Aoki et al. ${ }^{38}$

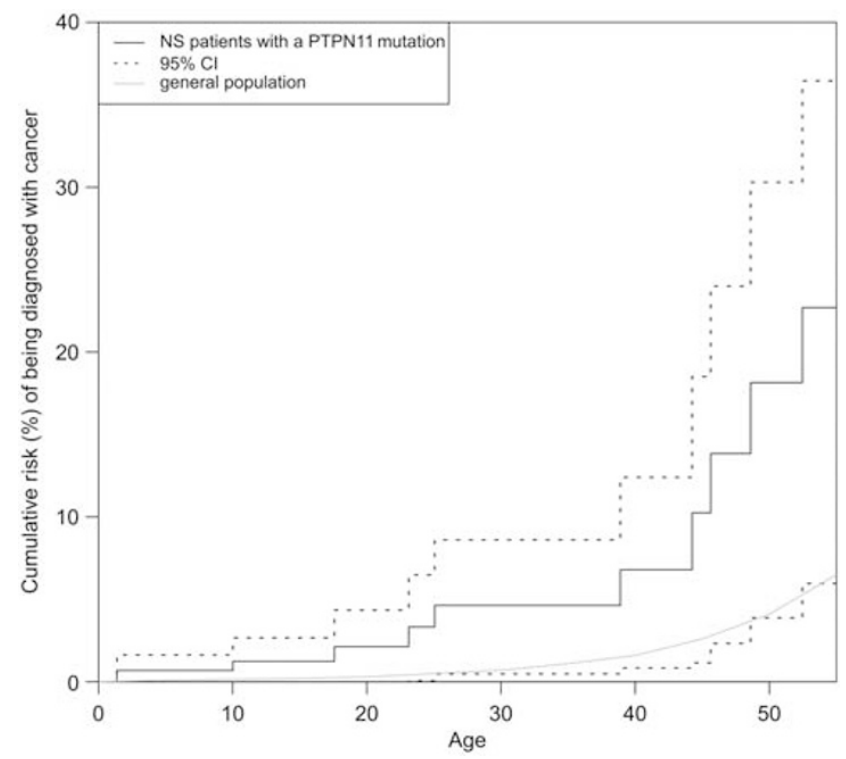

Figure 1 Age-specific cumulative risk of cancer in patients with Noonan syndrome and a mutation in PTPN11. We found a cumulative risk of $23 \%$ $(95 \% \mathrm{Cl}, 7.6-38.4 \%)$ up to age of 55 years, which represents a 3.5 -fold increased risk (95\% Cl, 2.0-5.9) compared with the general population.

signaling, are at a moderately increased risk of developing breast cancer. ${ }^{43}$ However, in a large panel of human breast cancers, no somatic mutations in PTPN11 were detected. ${ }^{19}$

It is known that somatic PTPN11 mutations associated with cancer generally confer a higher activation of SHP-2 function than those associated with NS. Likewise, SHP-2 mutants related to NS-associated leukemia exhibit higher PTP activity than do those related to NS alone. ${ }^{22,29,44}$ On the basis of these findings we hypothesized that the PTPN11 mutations found in our cohort of NS patients with a malignancy might show overlap with mutations detected somatically in cancer. Indeed, half of the NS patients with cancer are carriers of a mutation that has previously been reported as a somatic mutation in sporadic malignancies (Table 2). ${ }^{38}$ However, also $34 \%$ of the NS patients without cancer diagnosis in our cohort carried a mutation that has previously been detected somatically in cancer. These data suggest that assessment of the cancer risk based on the type of mutation detected is not feasible.

Remarkably, the p.Gly503Ala change identified in a patient with NS and JMML, has so far only been observed as a somatic mutation in JMML. For such mutations, which only seem to have a role as an acquired anomaly in leukemia, an association with embryonic lethality has been suggested. ${ }^{24} \mathrm{~A}$ mosaicism for the p.Gly503Ala mutation could explain our finding of this aberration in a life-born child. Unfortunately, no other tissues of this patient were available for further analysis.

As $65(22 \%)$ of the patients in the study cohort were born before PALGA and NCR had an almost complete coverage in the Netherlands, relevant data may be lacking for the first years of life of those patients. This may have resulted in an underestimation of pediatric cancers. On the other hand, as a result of the awareness of a tumor predisposition in NS among physicians, patients with NS and a malignancy are probably more likely to be referred for PTPN11 analysis than those with NS alone. This may have resulted in an overestimation of the life-time risk of cancer. Other constraints of our study are its retrospective nature and the young age distribution of the study cohort, with a median age of 13 years. Clearly, multicenter lifelong follow-up studies are required to evaluate the full spectrum of cancer risk in NS patients with a PTPN11 mutation. Similarly, such studies may be required to monitor the effect of growth hormone (GH) therapy for short stature in children with NS. As GH can cause proliferation of normal and malignant cells, the possibility that $\mathrm{GH}$ can induce cancer, specifically in persons with a tumor predisposition, has resulted in concerns over its use. Until now, however, follow-up studies in large cohorts of children and in smaller cohorts of adults have indicated that $\mathrm{GH}$ is not associated with an increased risk of tumor occurrence or recurrence (reviewed in refs Jenkins et al ${ }^{45}$; Ogilvy-Stuart and Gleeson ${ }^{46}$ ). But, as these studies have been performed in recipients of GH therapy who do not have a cancer predisposing genetic condition and the average duration of follow-up is only 4 years, we still recommend that all NS patients who have received GH should be included in follow-up programs to assess a possible relation between cancer risk and GH therapy in this specific condition.

A surveillance program for patients with a tumor predisposition syndrome is indicated when the benefits of such a strategy outweigh the disadvantages. The increased risk for developing a malignancy should be evident and early detection of cancer should positively influence patient outcome. In this study we showed that, although there is an increased risk of developing cancer in patients with NS with a PTPN11 mutation, the malignancies involve multiple sites and develop throughout life, making a straightforward screening protocol ineffective. Moreover, for hematological malignancies, most frequently occurring in NS, benefit of monitoring is unclear. Therefore, we conclude that at present a tumor surveillance program is not warranted for patients with NS carrying a PTPN11 mutation.

In conclusion, we have found an excess risk of cancer in patients carrying a mutation in PTPN11, compared with the risk of cancer in the general population. In addition to several malignancies that one would expect in a cohort of PTPN11 mutation carriers, some cancers were encountered that so far were not associated with NS. The initiation and/or progression of these tumors may very well be because of activating mutations in the PTPN11 gene. Both, on the basis of literature data and those presented here, however, no clear correlation has emerged between specific mutations in PTPN11 and the occurrence of cancer. Our data do not warrant a tumor surveillance program for patients with NS and a mutation in PTPN11. 


\section{CONFLICT OF INTEREST}

The authors declare no conflict of interest.

\section{ACKNOWLEDGEMENTS}

Marjolijn Jongmans is a MD-medical research trainee supported by The Netherlands Organization for Health Research and Development.

1 Tartaglia M, Zampino G, Gelb BD: Noonan syndrome: clinical aspects and molecular pathogenesis. Mol Syndromol 2010; 1: 2-26.

2 Choong K, Freedman MH, Chitayat D, Kelly EN, Taylor G, Zipursky A: Juvenile myelomonocytic leukemia and Noonan syndrome. J Pediatr Hematol Oncol 1999; 21: 523-527.

3 Lauchle JO, Braun BS, Loh ML, Shannon K: Inherited predispositions and hyperactive Ras in myeloid leukemogenesis. Pediatr Blood Cancer 2006; 46: 579-585.

4 Tartaglia M, Niemeyer CM, Shannon KM, Loh ML: SHP-2 and myeloid malignancies. Curr Opin Hematol 2004; 11: 44-50.

5 Chantrain CF, Jijon P, De RT et al: Therapy-related acute myeloid leukemia in a child with Noonan syndrome and clonal duplication of the germline PTPN11 mutation. Pediatr Blood Cancer 2007; 48: 101-104.

6 Ferrero GB, Baldassarre G, Delmonaco AG et al: Clinical and molecular characterization of 40 patients with Noonan syndrome. Eur J Med Genet 2008; 51: 566-572.

7 Karow A, Steinemann D, Gohring G et al: Clonal duplication of a germline PTPN11 mutation due to acquired uniparental disomy in acute lymphoblastic leukemia blasts from a patient with Noonan syndrome. Leukemia 2007; 21: 1303-1305.

8 La Starza R, Rosati R, Roti G et al: A new NDE1/PDGFRB fusion transcript underlying chronic myelomonocytic leukaemia in Noonan Syndrome. Leukemia 2007; 21: 830-833.

9 Lo FS, Kuo TT, Wang CJ, Kuo MT, Kuo MC: Hodgkin's lymphoma in a patient with Noonan syndrome with germ-line PTPN11 mutations. Int J Hematol 2008; 88: 287-290.

10 Matsubara K, Yabe H, Ogata T, Yoshida R, Fukaya T: Acute myeloid leukemia in an adult Noonan syndrome patient with PTPN11 mutation. Am J Hematol 2005; 79: 171-172.

11 Roti G, La SR, Ballanti S et al: Acute lymphoblastic leukaemia in Noonan syndrome. Br J Haematol 2006; 133: 448-450.

12 Cotton JL, Williams RG: Noonan syndrome and neuroblastoma. Arch Pediatr Adolesc Med 1995; 149: 1280-1281.

13 Ijiri R, Tanaka Y, Keisuke K, Masuno M, Imaizumi K: A case of Noonan's syndrome with possible associated neuroblastoma. Pediatr Radiol 2000; 30: 432-433.

14 Jung A, Bechthold S, Pfluger T, Renner C, Ehrt O: Orbital rhabdomyosarcoma in Noonan syndrome. J Pediatr Hematol Oncol 2003; 25: 330-332.

15 Khan S, McDowell H, Upadhyaya M, Fryer A: Vaginal rhabdomyosarcoma in a patient with Noonan syndrome. J Med Genet 1995; 32: 743-745.

16 Kondoh T, Ishii E, Aoki $Y$ et al: Noonan syndrome with leukaemoid reaction and overproduction of catecholamines: a case report. Eur J Pediatr 2003; 162: 548-549.

17 Moschovi M, Touliatou V, Papadopoulou A, Mayakou MA, Nikolaidou-Karpathiou P, Kitsiou-Tzeli S: Rhabdomyosarcoma in a patient with Noonan syndrome phenotype and review of the literature. J Pediatr Hematol Oncol 2007; 29: 341-344.

18 Mutesa L, Pierquin G, Janin N et al: Germline PTPN11 missense mutation in a case of Noonan syndrome associated with mediastinal and retroperitoneal neuroblastic tumors. Cancer Genet Cytogenet 2008; 182: 40-42.

19 Bentires-Alj M, Paez JG, David FS et al: Activating mutations of the Noonan syndromeassociated SHP2/PTPN11 gene in human solid tumors and adult acute myelogenous leukemia. Cancer Res 2004; 64: 8816-8820.

20 Fryssira H, Leventopoulos G, Psoni S, Kitsiou-Tzeli S, Stavrianeas N, Kanavakis E: Tumor development in three patients with Noonan syndrome. Eur J Pediatr 2008; 167: 1025-1031.

21 Lee JS, Tartaglia M, Gelb BD et al: Phenotypic and genotypic characterisation of Noonan-like/multiple giant cell lesion syndrome. J Med Genet 2005; 42: e11.

22 Tartaglia M, Martinelli S, Stella L et al: Diversity and functional consequences of germline and somatic PTPN11 mutations in human disease. Am J Hum Genet 2006; 78: 279-290.
23 Loh ML, Vattikuti S, Schubbert S et al: Mutations in PTPN11 implicate the SHP-2 phosphatase in leukemogenesis. Blood 2004; 103: 2325-2331.

24 Tartaglia M, Niemeyer CM, Fragale A et al: Somatic mutations in PTPN11 in juvenile myelomonocytic leukemia, myelodysplastic syndromes and acute myeloid leukemia. Nat Genet 2003; 34: 148-150.

25 Tartaglia M, Martinelli S, lavarone I et al: Somatic PTPN11 mutations in childhood acute myeloid leukaemia. Br J Haematol 2005; 129: 333-339.

26 Miyamoto D, Miyamoto M, Takahashi A et al: Isolation of a distinct class of gain-offunction SHP-2 mutants with oncogenic RAS-like transforming activity from solid tumors. Oncogene 2008; 27: 3508-3515.

27 Casparie M, Tiebosch AT, Burger G et al: Pathology databanking and biobanking in The Netherlands, a central role for PALGA, the nationwide histopathology and cytopathology data network and archive. Cell Oncol 2007; 29: 19-24.

28 Kratz CP, Niemeyer CM, Castleberry RP et al: The mutational spectrum of PTPN11 in juvenile myelomonocytic leukemia and Noonan syndrome/myeloproliferative disease. Blood 2005; 106: 2183-2185.

29 Niihori T, Aoki $\mathrm{Y}$, Ohashi $\mathrm{H}$ et al: Functional analysis of PTPN11/SHP-2 mutants identified in Noonan syndrome and childhood leukemia. J Hum Genet 2005; 50: 192-202.

30 Bader-Meunier B, Tchernia G, Mielot F et al: Occurrence of myeloproliferative disorder in patients with Noonan syndrome. J Pediatr 1997; 130: 885-889.

31 Silvio F, Carlo L, Elena B, Nicoletta B, Daniela F, Roberto M: Transient abnormal myelopoiesis in Noonan syndrome. J Pediatr Hematol Oncol 2002; 24: 763-764.

32 Lavin VA, Hamid R, Patterson J, Alford C, Ho R, Yang E: Use of human androgen receptor gene analysis to aid the diagnosis of JMML in female noonan syndrome patients. Pediatr Blood Cancer 2008; 51: 298-302.

33 Sherman CB, li-Nazir A, Gonzales-Gomez I, Finlay JL, Dhall G: Primary mixed glioneuronal tumor of the central nervous system in a patient with noonan syndrome: a case report and review of the literature. J Pediatr Hematol Oncol 2009; 31: 61-64.

34 Martinelli S, Carta C, Flex E et al: Activating PTPN11 mutations play a minor role in pediatric and adult solid tumors. Cancer Genet Cytogenet 2006; 166: 124-129.

35 Schuettpelz LG, McDonald S, Whitesell $\mathrm{K}$ et al: Pilocytic astrocytoma in a child with Noonan syndrome. Pediatr Blood Cancer 2009; 53: 1147-1149.

36 Shaw AC, Kalidas K, Crosby AH, Jeffery S, Patton MA: The natural history of Noonan syndrome: a long-term follow-up study. Arch Dis Child 2007; 92: 128-132.

37 Yoshida R, Ogata T, Masawa N, Nagai T: Hepatoblastoma in a Noonan syndrome patient with a PTPN11 mutation. Pediatr Blood Cancer 2008; 50: 1274-1276.

38 Aoki Y, Niihori T, Narumi Y, Kure S, Matsubara Y: The RAS/MAPK syndromes: novel roles of the RAS pathway in human genetic disorders. Hum Mutat 2008; 29: 992-1006.

39 Jongmans M, Sistermans EA, Rikken A et al: Genotypic and phenotypic characterization of Noonan syndrome: new data and review of the literature. Am J Med Genet $A$ 2005; 134A: 165-170.

40 Jongmans MC, Hoogerbrugge PM, Hilkens L et al: Noonan syndrome, the SOS1 gene and embryonal rhabdomyosarcoma. Genes Chromosomes Cancer 2010; 49: 635-641.

41 Vogelstein B, Fearon ER, Hamilton SR et al: Genetic alterations during colorectal-tumor development. N Engl J Med 1988; 319: 525-532.

42 Bentires-Alj M, Gil SG, Chan R et al: A role for the scaffolding adapter GAB2 in breast cancer. Nat Med 2006; 12: 114-121.

43 Sharif S, Moran A, Huson SM et al: Women with neurofibromatosis 1 are at a moderately increased risk of developing breast cancer and should be considered for early screening. J Med Genet 2007; 44: 481-484.

44 Keilhack H, David FS, McGregor M, Cantley LC, Neel BG: Diverse biochemical properties of Shp2 mutants. Implications for disease phenotypes. J Biol Chem 2005; 280: 30984-30993.

45 Jenkins PJ, Mukherjee A, Shalet SM: Does growth hormone cause cancer? Clin Endocrinol (Oxf) 2006; 64: 115-121.

46 Ogilvy-Stuart AL, Gleeson H: Cancer risk following growth hormone use in childhood: implications for current practice. Drug Saf 2004; 27: 369-382.

This work is licensed under the Creative Commons Attribution-NonCommercial-No Derivative Works 3.0 Unported Licence. To view a copy of this licence, visit http:// creativecommons.org/licenses/by-nc-nd/3.0/ 